\title{
First imported case of tick-borne encephalitis in Spain - was it
}

\section{alimentary?}

Camprubí Daniel $^{1 *}$, Moreno-García Estela², Almuedo-Riera Alex ${ }^{1}$, Martinez Mikel J11, 3 , Navarro Alexander ${ }^{3}$, Martinez-Hernandez Eugenia ${ }^{4}$, Muñoz Jose ${ }^{1}$, Ambrosioni Juan².

(1) ISGlobal, Hospital Clínic - Universitat de Barcelona, Barcelona, Spain.

(2) Infectious Diseases Department, Hospital Clínic de Barcelona, Barcelona, Spain.

(3) Microbiology Department, Hospital Clínic de Barcelona, Barcelona, Spain.

(4) Neurology Department, Hospital Clínic de Barcelona, Barcelona, Spain.

*Corresponding author: Daniel Camprubí

dcamprub@clinic.cat

Keywords: Tick-borne encephalitis, arbovirus, emerging infections. 


\section{First imported case of tick-borne encephalitis in Spain - was it}

\section{alimentary?}

Dear Editor,

As described by Kerlik, et al, tick-borne encephalitis (TBE) is an emerging infection in Europe and alimentary transmission is increasingly being reported in some European countries $[1,2]$. However, this is not the case in Spain where no previous cases have been reported [2,3]. Herein, we describe an imported case of TBE in Spain in a boy who probably acquired the virus by ingestion of contaminated milky products in a trip to Estonia.

An 18-year-old man with no previous medical history presented to the Emergency Room (ER) with high temperature and headache. He travelled to Hiiumaa, Estonia, where he had been working as a volunteer in a workcamp in a farm for three weeks, from $8^{\text {th }}$ to $29^{\text {th }}$ July 2019. During his stay in Estonia he participated in different farm activities such as taking care and milking sheep and goats and he consumed unpasteurized milk. He also reported that during the first week of his stay in the farm, three mates of the patient got ill, being diagnosed with TBE. The patient did not refer any tick bite and he had not been vaccinated against TBE before travelling.

On $28^{\text {th }}$ July, the patient presented with headache, weakness, myalgia, vomiting, walking imbalance and fever up to $39^{\circ} \mathrm{C}$. After 5 days, he consulted at the ER were meningeal 
signs were detected. Blood tests showed mild elevation of acute phase reactants, leukocytosis with neutrophilia, lymphopenia and thrombopenia. Under the clinical suspicion of acute meningitis, a lumbar puncture was performed, revealing lymphocytic pleocytosis with hyperproteinorrachia and normal glucose concentration levels in cerebrospinal fluid (CSF) (see Fig. 1). Empirical treatment with beta-lactams and doxycycline was then started. Blood and CSF cultures resulted negative. A multiplex polymerase chain-reaction (PCR) on CSF sample was performed, allowing to discard common bacterial, viral and fungal causes of central nervous system infections. HIV serology and HIV viral load resulted negative. West-Nile virus RT-PCR, a flavivirus generic RT-PCR and Borrelia antibodies were tested in CSF and resulted negative. Serologies for Coxiella burnetii, Borrelia burgdorferi, Brucella spp., Rickettsia conorii, Leptospira spp. and Treponema pallidum were also negative.

A commercial immunofluorescence assay (Euroimmun, Lübeck, Germany) detecting antibodies against West Nile, Japanese encephalitis, yellow fever and TBE viruses was used in CSF and serum samples. Antibodies against TBE were detected in a CSF samples obtained 6 days after the onset of symptoms (d.o.s) at a low titer (1:4) with no crossreactivity observed against the other flaviviruses tested. Subsequent serum sample collected eight d.o.s tested negative against IgM and IgG against dengue (ELISA, Panbio Diagnostics) and against West Nile (ELISA, Focus Diagnostics) and positive for IgM (titer 1:80) and IgG (titer 1:320) against TBE, with only minimal cross reactivity observed in IgG against Japanese encephalitis (titer 1:20). In a convalescent sample obtained 31 d.o.s, IgG antibodies against TBE were positive at a titer of 1:2560 (see Fig. 1). The detection of TBE IgM antibodies in CSF, specific TBE IgM and IgG in serum, and the 8- 
folds increase of TBE IgG antibodies in paired serum samples fulfilled the European Union case definition criteria for a laboratory confirmed case of TBE [4].

Diagnosis of TBE, probably acquired by consumption of unpasteurized milky products [1], was then confirmed. Brain Magnetic Resonance Imaging (MRI) did not show any alteration. The patient presented a very good evolution, allowing to discontinue the antibiotic treatment and being discharged after 7 days of admission.

However, three weeks later the patient presented a mild non-disabling distal tremor on the right upper limb. A new brain MRI was unremarkable and anti-NMDAR antibodies resulted negative, allowing to rule out an auto-immune induced encephalitis. After three months, the patient was completely recovered, allowing him to return to his daily activities (see Fig. 1).

Tick-borne encephalitis (TBE) is an infection caused by a flavivirus, usually transmitted by Ixodes ticks. Concerning European countries, TBE mainly affects countries in central and eastern Europe. Interestingly, Ixodes ricinus is widely distributed through all Europe including Spain and seroprevalence studies in dogs reported circulation of TBE virus in Spain [5]. However, no human cases of TBE in Spain have been reported in peer-review journals. In fact, there was no surveillance system for TBE until 2013. From then on, no cases in humans have been neither reported in Spain in National or International Surveillance Reports [3]. Besides being transmitted through tick bites, TBE can also be acquired after consumption of unpasteurized contaminated milky products, what has 
been occasionally associated with TBE outbreaks, especially in some Central-Eastern European countries [1].

So far, we described an imported case of TBE in Spain in an 18-year-old boy who travelled to Estonia, who probably acquired the virus by ingestion of contaminated dairy products. Being tick-borne diseases one of the major concerns in emerging infections disease in Europe, surveillance of such entities is crucial. Moreover, infrequent transmission routes, such as in our case should be considered.

Our report highlights the importance of monitoring infections such as TBE to be aware of imported emerging infections such as arthropod-borne diseases, especially in nonendemic countries. On the other hand, pre-travel consultation and vaccination when indicated, play a key role in preventing cases like the one presented [6]. 


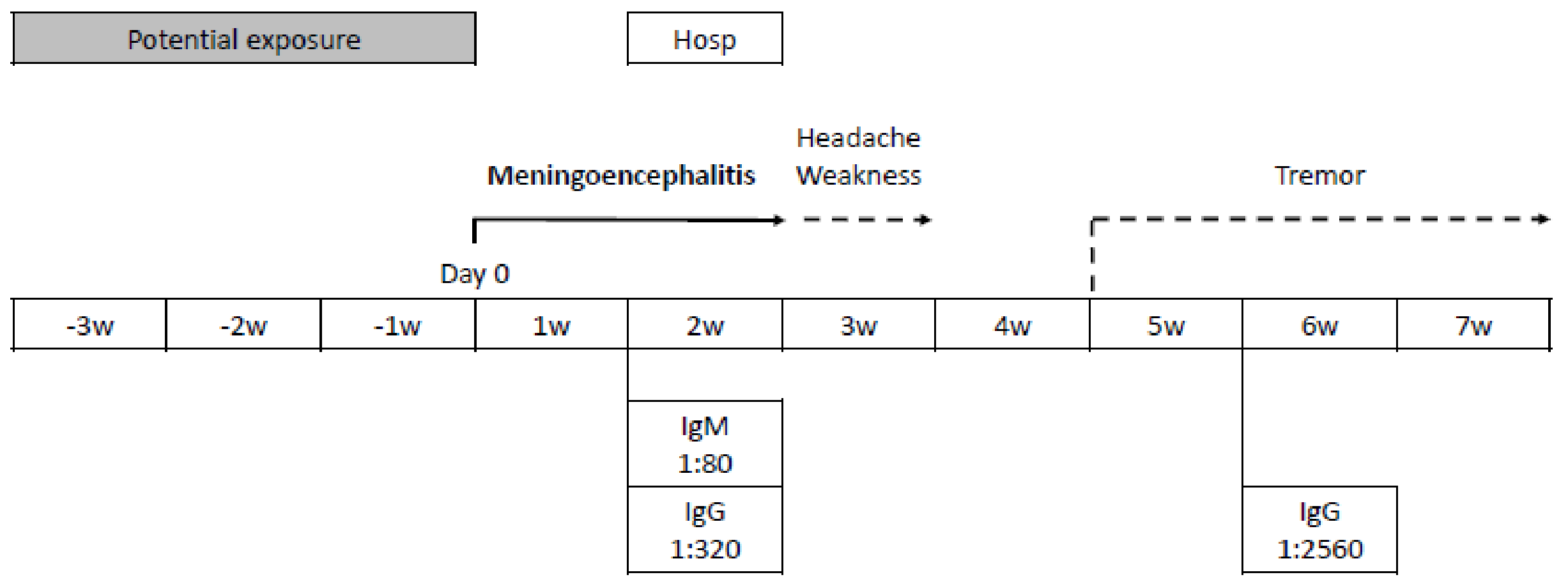

Figure - Chronology of symptoms and serological evolution of the case. Onset of symptoms (day 0). Timeline divided into weeks (lower squares). Hosp: hospitalization. IgM: Immunoglobulin M. IgG: Immunoglobulin G. 


\section{REFERENCES}

[1] Kerlik J, Avdičová M, Štefkovičová M, Tarkovská V, Pantiková M, Valachová P, Molčányi T, Mecencev R. Slovakia reports highest occurrence of alimentary tick-borne encephalitis in Europe: Analysis of tick-borne encephalitis outbreaks in Slovakia during 2007-2016. Travel Med Infect Dis. 2018; 26: 37-42.

[2] European Centre for Disease Prevention and Control. Tick-borne encephalitis. In: ECDC. Annual epidemiological report for 2017. Stockholm: ECDC; 2019.

[3] Departamento de Epidemiología, Comunidad de Madrid. Informe de Morbilidad por enfermedades de Declaración Obligatoria. Año 2017. p. 1-121.

https://www.comunidad.madrid/sites/default/files/doc/sanidad/epid/informe_edo_2 017.pdf

[4] Commission Implementing Decision (EU) 2018/945 of 22 June 2018 on the communicable diseases and related special health issues to be covered by epidemiological surveillance as well as relevant case definitions. Available at: https://eur-lex.europa.eu/legalcontent/EN/TXT/PDF/?uri=CELEX:32018D0945\&from=EN\#page $=45$

[5] I García-Bocanegra, E Jurado-Tarifa, D Cano-Terriza, R Martínez, JE Pérez-Marín, S Lecollinet. Exposure to West Nile virus and tick-borne encephalitis virus in dogs in Spain. Transbound Emerg Dis. 2018 Jun;65(3):765-772. doi: 10.1111/tbed.12801. 
[6] Steffen R. Epidemiology of tick-borne encephalitis (TBE) in international travellers to Western/Central Europe and conclusions on vaccination recommendations. Journal of Travel Medicine. 2016, 1-10. doi: 10.1093/jtm/taw018. 